\title{
Processing of Odor Mixtures in the Zebrafish Olfactory Bulb
}

\author{
Rico Tabor, Emre Yaksi, Jan-Marek Weislogel, and Rainer W. Friedrich \\ Department of Biomedical Optics, Max Planck Institute for Medical Research, D-69120 Heidelberg, Germany
}

Components of odor mixtures often are not perceived individually, suggesting that neural representations of mixtures are not simple combinations of the representations of the components. We studied odor responses to binary mixtures of amino acids and food extracts at different processing stages in the olfactory bulb $(\mathrm{OB})$ of zebrafish. Odor-evoked input to the $\mathrm{OB}$ was measured by imaging $\mathrm{Ca}^{2+}$ signals in afferents to olfactory glomeruli. Activity patterns evoked by mixtures were predictable within narrow limits from the component patterns, indicating that mixture interactions in the peripheral olfactory system are weak. OB output neurons, the mitral cells (MCs), were recorded extra- and intracellularly and responded to odors with stimulus-dependent temporal firing rate modulations. Responses to mixtures of amino acids often were dominated by one of the component responses. Responses to mixtures of food extracts, in contrast, were more distinct from both component responses. These results show that mixture interactions can result from processing in the OB. Moreover, our data indicate that mixture interactions in the OB become more pronounced with increasing overlap of input activity patterns evoked by the components. Emerging from these results are rules of mixture interactions that may explain behavioral data and provide a basis for understanding the processing of natural odor stimuli in the $\mathrm{OB}$.

Key words: olfaction; odor mixture; information processing; olfactory glomerulus; mitral cell; neural circuit

\section{Introduction}

Olfaction is considered a "synthetic" sense because the ability to segment the perception of odor mixtures into distinct components is limited (Laing and Francis, 1989; Livermore and Laing, 1996). The perception of an odor mixture either is dominated by an intense component or acquires a new quality (Moskowitz and Barbe, 1977; Laing and Willcox, 1983; Laing et al., 1984, 1989; Bell et al., 1987; Staubli et al., 1987; Derby et al., 1996; Valentincic et al., 2000; Wiltrout et al., 2003). Thus, responses to odorants are assumed to "interact" in the response to a mixture, giving rise to a neural representation that discards information about individual components but acquires mixture-specific properties. Such mixture interactions may be important for the processing of natural odor stimuli.

Chemicals are detected by olfactory sensory neurons (OSNs), each of which expresses one of $>1000$ odorant receptors (ORs) in rodents (Malnic et al., 1999; Mombaerts, 1999; Zhang and Firestein, 2002). OSNs expressing the same odorant receptor converge onto distinct olfactory glomeruli (Ressler et al., 1994; Vassar et al., 1994; Mombaerts, 1999). Odor quality initially is represented in the olfactory bulb (OB) by a combinatorial activity pattern across glomeruli, each of which responds to multiple odors (Stewart et

Received May 12, 2004; revised June 9, 2004; accepted June 10, 2004

This work was supported by the Max Planck Society, the Deutsche Forschungsgemeinschaft, and a fellowship from the Boehringer Ingelheim Fonds to E.Y. We thank Gilles Laurent for generous support and Hartwig Spors for comments on this manuscript.

Correspondence should be addressed to Dr. Rainer Friedrich, Max Planck Institute for Medical Research, Department of Biomedical Optics, Jahnstrasse 29, D-69120 Heidelberg, Germany. E-mail: Rainer.Friedrich@ mpimf-heidelberg.mpg.de.

J.-M. Weislogel's present address: Interdisciplinary Center for Neurosciences, University of Heidelberg, D-69120 Heidelberg, Germany.

DOI:10.1523/JNEUROSCI.1834-04.2004

Copyright $\odot 2004$ Society for Neuroscience $\quad$ 0270-6474/04/246611-10\$15.00/0 al., 1979; Lancet et al., 1982; Friedrich and Korsching, 1997; Rubin and Katz, 1999; Fuss and Korsching, 2001; Wachowiak and Cohen, 2001). The principal neurons of the OB, the mitral cells (MCs), receive input from OSNs and interact via local interneurons. Synaptic connections within the OB shape the tuning profiles of MCs and temporally pattern their responses.

Interactions between components in a mixture may occur at different stages of odor processing. In OSNs, mixture interactions have been observed in vertebrates and invertebrates and may be mediated by competitive inhibition or synergistic activation of odorant receptors or by cross-talking signal transduction pathways (Ache et al., 1988; Laing et al., 1989; Ache and Zhainazarov, 1995; Kang and Caprio, 1997; Cromarty and Derby, 1998; Spehr et al., 2002). Mixture interactions also have been observed in the OB (Kang and Caprio, 1995; Giraudet et al., 2002; Wilson, 2003); however, the origin of the interactions has remained unknown. A 2-deoxyglucose study suggested that mixture interactions occur primarily in the periphery (Bell et al., 1987), although evidence exists for central mixture interactions in the spiny lobster (Derby et al., 1985). It is therefore unclear how neural circuits in the brain contribute to the processing of odor mixtures. Moreover, understanding the processing of natural odor stimuli would be facilitated by a set of rules describing the effect of mixture interactions on odor-evoked activity patterns.

We examined binary mixture interactions in the $\mathrm{OB}$ of adult zebrafish, which is similar to that of other vertebrates but contains relatively few glomeruli and MCs (Baier and Korsching, 1994; Byrd and Brunjes, 1995; Edwards and Michel, 2002). Binary mixture interactions were studied in patterns of afferent glomerular activity and in $\mathrm{MC}$ odor responses, representing $\mathrm{OB}$ input and output activity, respectively. Significant interactions were observed in $\mathrm{MC}$ responses, but not their input activity patterns, indicating that the $\mathrm{OB}$ contributes to the processing of 
odor mixtures. Tentative rules for mixture interactions in the $\mathrm{OB}$ were derived and provide a basis for understanding the effect of mixture interactions on odor representations by multiple neurons.

\section{Materials and Methods}

Animals and loading of OSNs with a $\mathrm{Ca}^{2+}$ indicator. Adult zebrafish (Danio rerio) were obtained from a local supplier or from a laboratory colony and kept at $22-26^{\circ} \mathrm{C}$. Loading of OSNs with calcium green-1 dextran (10 kDa; Molecular Probes, Eugene, OR) was performed as described (Friedrich and Korsching, 1997). Briefly, $\sim 1 \mu \mathrm{l}$ of $6-8 \%$ calcium green- 1 dextran in $3 \mathrm{~mm} \mathrm{NaCl}$ and $0.1 \%$ Triton X-100 was applied into each naris under anesthesia with $0.01 \%$ MS-222. After 5 min the dye solution was washed away, and the fish were allowed to recover. Triton X-100 transiently permeabilized olfactory cilia, allowing uptake of the dye. Cilia regenerated during the next $48 \mathrm{hr}$ (Friedrich and Korsching, 1997). Fish were used for imaging experiments 3-6 d after dye loading.

Surgery and odor stimulation. An explant of the intact zebrafish brain and nose was used in all experiments and prepared as described (Friedrich and Laurent, 2001, 2004). Briefly, fish were cooled to $4^{\circ} \mathrm{C}$ and decapitated in teleost artificial CSF (ACSF) (Mathieson and Maler, 1988). The eyes, jaws, and bones over the ventral forebrain were removed to expose the ventral OBs. The preparation was placed ventral-side up into a custom-made flow chamber, continuously superfused with ACSF, and allowed to warm up to room temperature $\left(\sim 22^{\circ} \mathrm{C}\right)$. In some imaging experiments that used an inverted microscope, brain structures caudal to the optic chiasm were removed, and the preparation was placed ventralside down in a chamber with a coverslipped window in the floor as described (Friedrich and Korsching, 1997). All animal procedures were performed in accordance with the animal care guidelines issued by the Federal Republic of Germany.

Odors were delivered through a constant carrier stream directed at the ipsilateral inflow naris by a computer-controlled, pneumatically actuated HPLC injection valve (Valco, Houston, TX, or Rheodyne, Rohnert Park, $\mathrm{CA}$ ). The time course of the odor stimulus was measured by imaging the efflux of a fluorescein solution from the delivery tube. The stimulus rose to maximum concentration within $\sim 600 \mathrm{msec}$ and persisted for $\sim 2.4$ sec. The stimulus time course was highly reproducible. Amino acids (Sigma, St. Louis, MO, or Fluka, Neu-Ulm, Germany) were of the highest purity available. Fresh stock solutions ( 1 or $10 \mathrm{~mm}$ ) were prepared at least every $10 \mathrm{~d}$, refrigerated, and diluted to final concentrations of $10-40 \mu \mathrm{M}$ immediately before the experiment. Food extracts were prepared from commercially available flake food or from commercially available dried tubifex, daphnia, or blood worms. Stock solutions were made at least every $14 \mathrm{~d}$ by incubating $200 \mathrm{mg}$ of fish food in $50 \mathrm{ml}$ of ACSF for $1 \mathrm{hr}$ and filtering through filter paper. Stock solutions were kept refrigerated and diluted 1:10 to 1:100 immediately before the experiment. The dilution factor was chosen so that the peak $\mathrm{Ca}^{2+}$ signals evoked by food extracts and amino acid stimuli were similar. Seven different foods were used. In all experiments the concentration of each component was the same when applied alone or in the mixture. Odor applications were separated by at least $105 \mathrm{sec}$ to exclude sensory adaptation. Stimuli did not saturate glomerular responses because higher concentrations evoked larger $\mathrm{Ca}^{2+}$ signals in most glomeruli (Friedrich and Korsching, 1997).

$\mathrm{Ca}^{2+}$ imaging. Specimens were viewed either with an inverted microscope (Zeiss Axiovert 100, Oberkochen, Germany) equipped with a $10 \times$ air objective [numerical aperture (NA) 0.5 ; Zeiss] or with a custom-made upright epifluorescence microscope that used a BX-RFA epifluorescence condenser (Olympus, Tokyo, Japan) and a $20 \times$ objective (NA 0.95; Olympus). Fluorescence was excited with a $150 \mathrm{~W}$ xenon arc lamp equipped with a stabilized power supply (Opti Quip, Highland Mills, NY) through an excitation filter $(495 / 30 \mathrm{HQ})$. Light was attenuated to $1.5 \%$ of the full intensity by neutral density filters to minimize photobleaching. Emitted light was projected through a dichroic mirror (Q520LP) and emission filter (HQ545/50) onto the chip of a CCD camera (CoolSnapHQ, Photometrics, Tucson, AZ). Images were binned to a final resolution of $130 \times 174$ pixels and digitized at 12 bits and $2-16 \mathrm{~Hz}$. Each pixel value in an image series was converted to a value representing the relative change in fluorescence $(\Delta F / F)$ after stimulus application. The baseline fluorescence $(F)$ was calculated by averaging over the frames before stimulus onset. No bleach correction was performed, because bleaching was minimal and a correction would introduce additional noise. Response maps were obtained by averaging $\Delta F / F$ frames over $2-4$ sec after response onset and mild spatial filtering (Gaussian filter of $\sigma=$ 1.2 pixels; width, 5 pixels). The signal-to-noise ratio was substantially higher in experiments performed with the upright microscope and the $20 \times$, NA 0.95 objective. Quantitative analysis therefore was restricted to these experiments $(n=35)$. However, results obtained with the inverted microscope ( $n=22$ experiments) were qualitatively indistinguishable.

Electrophysiology. Axoclamp 2B amplifiers (Axon Instruments, Foster City, CA) in bridge mode were used for all recordings. Intracellular whole-cell patch-clamp recordings $(n=13$ cells) were performed with borosilicate pipettes (12-18 M 2 ). The pipette solution contained (in $\mathrm{mm}) 130 \mathrm{~K}$-gluconate, $10 \mathrm{Na}$-gluconate, $10 \mathrm{Na}$-phosphocreatine, $4 \mathrm{NaCl}$, 4 Mg-ATP, $0.3 \mathrm{Na}$-GTP, 10 HEPES, pH 7.25. Mitral cells in the superficial layers were approached under visual control by using differential interference contrast (DIC) optics and contrast-enhanced video display. Extracellular recordings were performed in the loose-patch or cell-attached configuration. In cell-attached recordings $(n=7)$ the pipettes (12-18 M $\Omega$ ) were filled with intracellular solution, and MCs were approached under visual control. Loose-patch recordings $(n=32)$ were done as described (Friedrich and Laurent, 2001, 2004) by using pipettes filled with ACSF $(9-12$ $\mathrm{M} \Omega$ ). Results obtained with the three different recording methods were indistinguishable. In total, responses to 57 stimulus sets (components and mixture) were recorded from $52 \mathrm{MCs}$. The maximum number of stimulus sets tested on a single MC was three. Each odor stimulus was repeated, on average, $6 \pm 3$ times. Responses of the same neuron to the same stimulus were averaged in peristimulus time histograms (PSTHs) with $100 \mathrm{msec}$ bin width. A stimulus presented early during the experiment usually was repeated at a later time to ensure the stability of responses (Friedrich and Laurent, 2004), or stimuli were interleaved.

Prediction of the mixture response from $\mathrm{Ca}^{2+}$ imaging data. Presynaptic glomerular $\mathrm{Ca}^{2+}$ signals were, with very few exceptions, positive-going and increased monotonically with stimulus concentration until saturation (Friedrich and Korsching, 1997). Because of these properties, the algorithm explained in Figure 1 was used to predict mixture responses from the component responses. Often, relatively large regions in the activity map showed no detectable odor response. To minimize the influence of the noise in these regions on the quantitative analysis, pixel values of $\Delta F / F<0.4 \%$ were set to zero and excluded from quantitative analysis. This threshold corresponds to $\sim 2 \mathrm{SDs}$ of the average pixel noise $(0.21 \pm 0.07 \% \Delta F / F ; n=35$ experiments). The average pixel noise was determined in subtractions of two response maps measured in the absence of odor stimulation.

When the response to one component is below response threshold or saturating, the mixture response would be expected to be equal to the response to the more potent component alone; i. e., the superposition term (see Fig. 1) should be zero. This is achieved by using the geometric mean in the calculation of the superposition term. The disadvantage is, however, that the geometric mean can rectify noise; when no real response increase occurs in a pixel between 1 and $2 \times$ concentration (because the stimulus is below threshold or saturating), noise can lead to small negative difference values in the terms $\Delta_{2 \times-1 \times}$ Resp OdorX $_{\text {(see Fig. }}$ 1). The multiplication then yields a positive value, thereby biasing the prediction to more positive values. This effect is relatively small and occurs primarily in nonresponsive regions. Nevertheless, to avoid this,

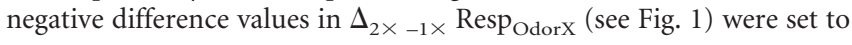
zero.

Statistical comparisons. All statistical comparisons were performed by using a nonparametric Wilcoxon rank sum test. All given error values are SDs.

\section{Results}

\section{Measurement of odor responses in sensory afferents to the $\mathrm{OB}$}

Odor-evoked activity in sensory afferents to glomeruli was measured by $\mathrm{Ca}^{2+}$ imaging after OSNs were loaded with calcium 
green-1 dextran (Friedrich and Korsching, 1997). Experiments were performed in a ventrolateral region of the OB that contains small, densely packed glomerular structures responding preferentially to amino acids and nucleotides (Friedrich and Korsching, 1997, 1998). Odor application to the nose evokes distinct patterns of fluorescence signals in the $\mathrm{OB}$, reflecting changes in $\mathrm{Ca}^{2+}$ concentration in OSN axonal terminals (Friedrich and Korsching, 1997; Fuss and Korsching, 2001; Wachowiak and Cohen, 2001; Wachowiak et al., 2004) (see Fig. 2A1-A4). Application of the carrier solution alone did not evoke detectable signals (data not shown). As shown previously (Friedrich and Korsching, 1997), the time courses of odor-evoked $\mathrm{Ca}^{2+}$ signals were similar and stereotyped throughout the observed region. This is consistent with odor responses of single zebrafish OSNs recorded electrophysiologically: OSN responses follow a stereotyped phasictonic time course, and the pattern of activity across multiple OSNs does not change much during odor stimulation (Friedrich and Laurent, 2001, 2004). Maps of $\mathrm{Ca}^{2+}$ signals, therefore, were time averaged. Negative-going responses were very rare, consistent with the low abundance of inhibitory electrical responses in zebrafish OSNs (Friedrich and Laurent, 2004). Responses of glomerular units have odor- and glomerulus-specific thresholds and increase monotonically with concentration over several log units until saturation (Friedrich and Korsching, 1997).

\section{Analysis of mixture interactions in sensory afferents to the $\mathrm{OB}$}

The monotonicity of concentration-response functions and the very rare occurrence of negative-going responses are reminiscent of concentration-response curves describing ligand-receptor interactions. We therefore used a simple algorithm based on models of ligand-receptor activation to predict afferent patterns of $\mathrm{Ca}^{2+}$ signals evoked by binary odor mixtures from the patterns evoked by their components (Fig. 1). This algorithm assumes that components (ligands) do not interact and activate a common effector (receptor) associated with each glomerulus. The strength of activation of a given glomerulus by a given ligand is described by the corresponding concentration-response function. Local information about concentration-response functions was obtained by measuring responses to components at the concentration used in the mixture $(1 \times)$ and at twice that concentration $(2 \times)$. The minimum signal expected in response to the mixture is the signal elicited by the more potent component at the $1 \times$ concentration $\left(\operatorname{Max}_{1 \times}\right.$; Fig. 1). Added to this signal is the signal increment expected from the second component (Superposition term; Fig. 1). This is approximated as the geometric mean of the signal increase between $1 \times$ and $2 \times$ concentrations for each component. The geometric mean was used because it yields correct values when the concentration of one component is below response threshold or saturating (i.e., when the concentration-response function has a slope near zero; see Fig. 1 and Materials and Methods). The calculation yields three values: a conservative lower bound $\left(\operatorname{Max}_{1 \times}\right)$, a conservative upper bound $\left(\operatorname{Max}_{2 \times}\right.$; the signal elicited by the more potent component at $2 \times$ concentration in each pixel), and the prediction (Fig. 1). The difference between lower and upper bounds is usually small relative to the absolute response magnitude, because a twofold concentration change is small relative to the dynamic range and affects activity patterns only slightly (see below). The prediction error arising from uncertainties in the concentration-response function therefore is also small. Nevertheless, the prediction by the geometric mean is an approximation; precise knowledge of the concentration-response function would be required to construct an
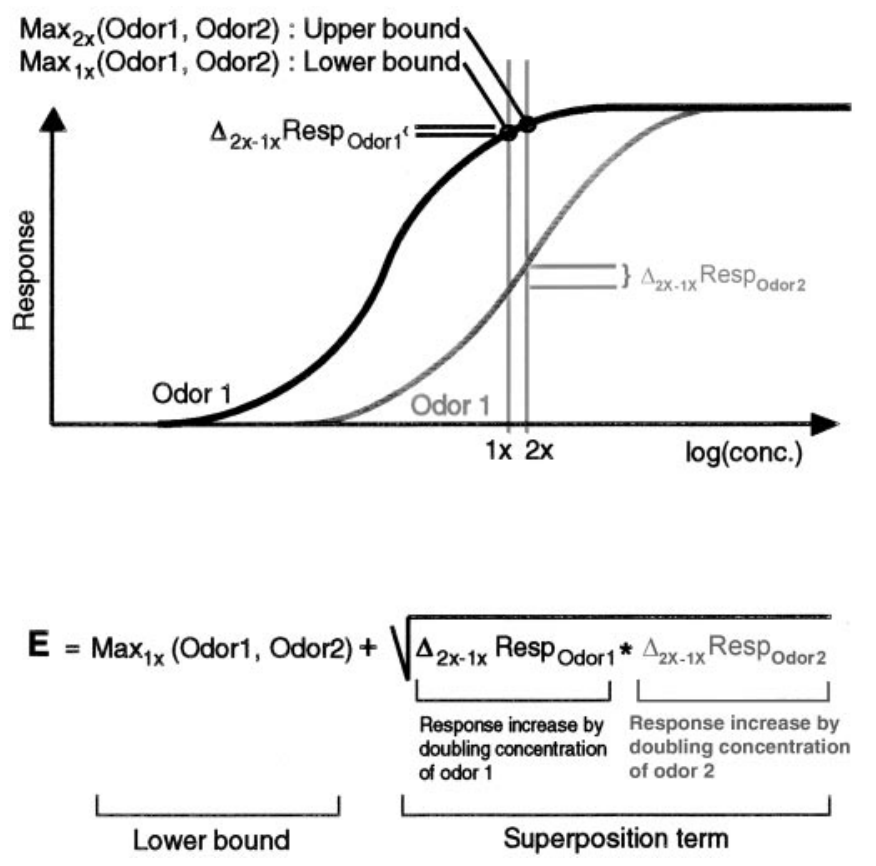

Figure 1. Schematic illustration of the algorithm for prediction of afferent activity patterns evoked by a binary odor mixture. The algorithm assumes that both components stimulate the same effector site with different potency and potentially also with different concentrationresponse functions. The algorithm requires that negative responses do not occur and that concentration-response functions are monotonic. Information about the full concentration-response functions is not required, but information about the local slope of the functions is sufficient. This information was obtained by applying each component of a binary mixture alone at the concentration used in the mixture $(1 X)$ and at twice that concentration $(2 X)$. In each pixel a lower bound for the expected response to the mixture is given by the larger of the $\Delta F / F$ values evoked by the two components at $1 \times$ concentration, $\operatorname{Max}_{1 \times}$ (0dor1, 0dor2), whereas an upper bound is given by the larger of the $\Delta F / F$ values evoked by the two components at $2 X$ concentration, $\operatorname{Max}_{2 \times}$ (0dor1, 0dor2). The prediction is calculated by adding a "superposition term" to the lower bound. The superposition term represents the expected signal increase over the lower bound because of the second component. It is calculated as the geometric mean of the increase in $\mathrm{Ca}^{2+}$ signal between $1 \times$ and $2 \times$ concentration for each odor $\left(\Delta_{2 \times-1 \times}\right.$ Resp $p_{0 \text { dor } 1}$ and $\Delta_{2 \times-1 \times}$ Resp $\left.p_{0 \text { dor } 2}\right)$. Using the geometric mean ensures that the algorithm performs correctly when the slope of the concentration-response function for one or both odors is near zero. Nevertheless, the calculated prediction is an approximation, rather than a precise calculation, of the expected signal in response to the mixture.

exact algorithm. Moreover, the calculation of the prediction accumulates noise in the data, arising, for example, from shot noise and the low amount of bleaching. To minimize these effects, we included only those pixels in which the response $(\Delta F / F)$ was $>0.4 \%$ in the analysis.

We first examined glomerular $\mathrm{Ca}^{2+}$ signals in response to single amino acids, which are natural odors for fish and other aquatic animals. Concentrations used were $10-40 \mu \mathrm{M}$, which are intermediate in the behaviorally relevant range (Carr, 1988). Figure $2 A 1-A 4$ shows $\mathrm{Ca}^{2+}$ signal maps evoked by two amino acid components at 1 and $2 \times$ concentrations in the ventrolateral $\mathrm{OB}$, thresholded at $\Delta F / F=0.4 \%$. From these responses the bounds and the prediction were calculated pixel-wise and compared with the actual mixture response.

The lower and upper bounds (Fig. 2A5,A6) were only slightly different from each other. The prediction (Fig. 2A7) was very similar to the measured mixture response (Fig. 2A8). Responsive regions in the pattern evoked by the mixture corresponded to those in the prediction, and similar distributions of response magnitude were observed in the two maps. The sum of the component patterns (Fig. 2A9) did not predict response patterns as 
well. This was expected, because it did not take into account the nonlinearity of concentration-response functions.

Figure 2 A10 depicts the difference between the mixture response and the prediction. Negative and positive deviations from zero were encoded by opposite colors. Deviations were small relative to the overall response magnitude (note different calibration of color scales) and appeared to be only slightly greater than deviations between maps evoked by repeated applications of the mixture (Fig. 2A11). Similar results were obtained in $n=33$ experiments.

The deviation between the mixture response and the prediction was quantified in 18 experiments performed under conditions of low photon noise (see Materials and Methods). The differences in pixel values between the prediction and the actual mixture response are shown as histograms in Figure 2C. Similar distributions were measured in two consecutive series of applications (Fig. 2C, blue and cyan, respectively), indicating that responses were stable. The widths of these distributions, quantified by the SD of a Gaussian fit to the histogram, were not significantly different from each other ( $p>0.65$; Fig. $2 D$ ). These distributions were slightly but significantly broader than the distribution of difference values for repeated mixture responses (Fig. 2C, red, D). Activity maps evoked by repeated responses to the mixture were therefore more similar to each other than to the prediction. These results were confirmed by using the correlation coefficient as a measure of similarity. The mean correlation between the prediction and the mixture response was $0.89 \pm 0.06$ for the first series of stimuli and $0.88 \pm$ 0.09 for the second set of stimuli (not significantly different; $p>0.8$ ), whereas the mean correlation between maps evoked by repeated mixture applications was slightly but significantly higher $(0.93 \pm 0.06$; $p<$ $0.05)$.

The slightly higher similarity between repeated mixture responses could indicate a significant deviation of the prediction from the mixture response. However, it is likely that this deviation is at least partially a consequence of the calculation of the prediction, because the prediction is not exact and because the procedure accumulates noise. Moreover, the correlation between mixture responses and prediction was high, and the average difference between the SDs of the Gaussian fits was $0.05 \pm 0.03 \%$ $\Delta F / F$. This value is very small compared with the $\Delta F / F$ values in the response maps. We therefore conclude that afferent glomerular responses to binary mixtures of amino acids are predictable within narrow limits by a model assuming no interactions between components.
A

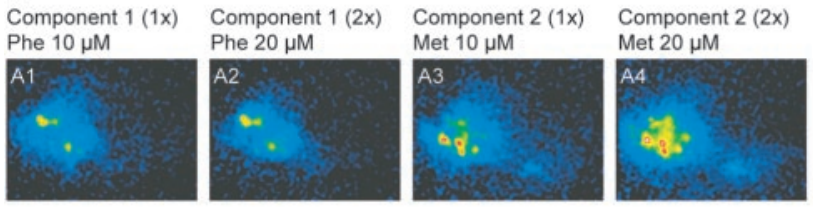

Lower bound Upper bound Highest pix. val. 1x

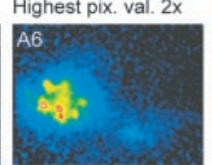

Prediction
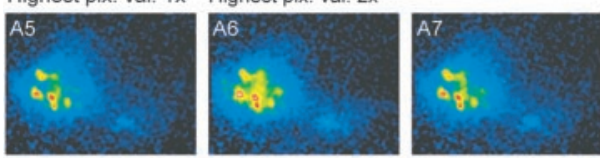

Mixture

Phe + Met
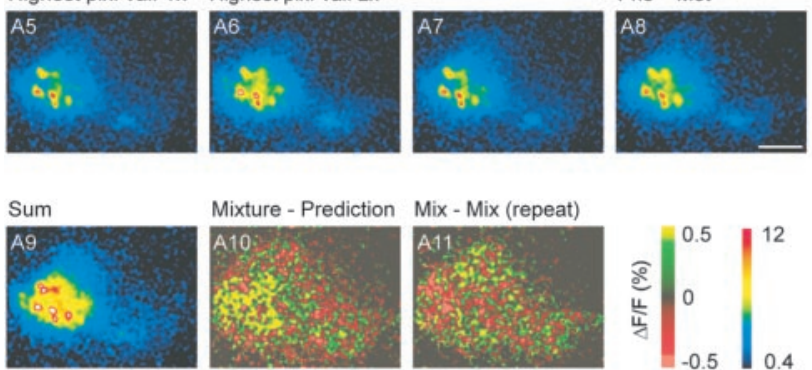

Mixture - Prediction Mix - Mix (repeat)
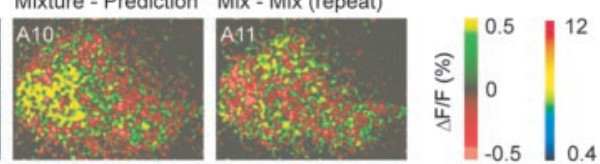

$\mathbf{B}$
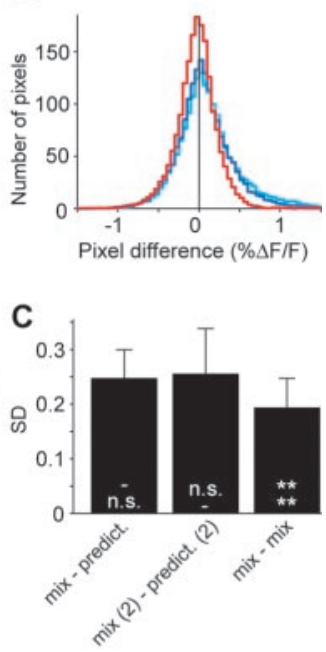

D

Component 1 1 ( $\mathrm{x}) \quad$ Component $1(2 \mathrm{x}) \quad$ Component $2(1 \mathrm{x}) \quad$ Component $2(2 \mathrm{x})$ Food ex. 3 1:100 dil. Food ex. $31: 50$ dil. Food ex. 5 1:100 dil. Food ex. 5 1:50 dil.
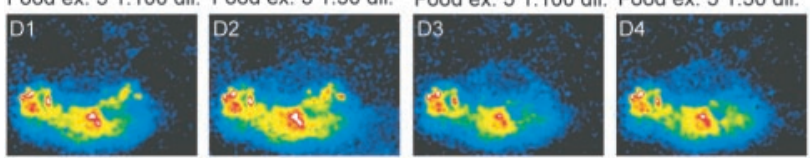

Lower bound

Upper bound

Highest pix. val. 1x

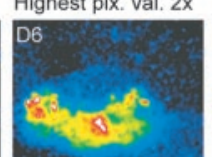

Prediction

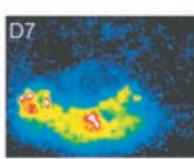

Mixture

F. ex. $3+$ F. ex. 5
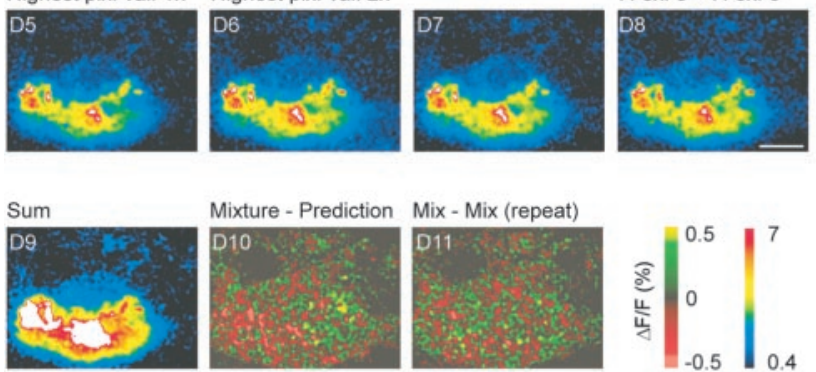

E

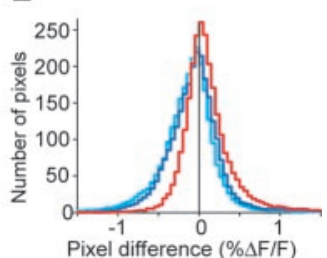

$\mathbf{F}$

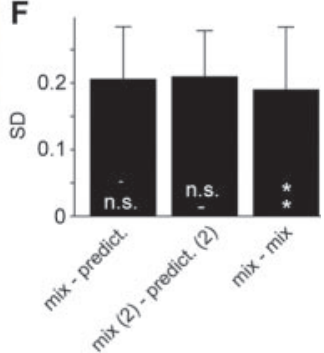

Figure 2. Patterns of afferent glomerular activity evoked by binary odor mixtures and their components. A1-A4, Maps of afferent glomerular $\mathrm{Ca}^{2+}$ signals in the ventrolateral $O B$, evoked by two amino acid components at $1 \times$ and $2 \times$ concentration. Lateral is to the bottom; anterior is to the right. $A 5-A 7$, Lower bound, upper bound, and prediction (see Fig. 1) as calculated from $A 1-A 4$. A8, Measured response to the mixture. A9, Sum of component activity patterns at $1 \times$ concentration $(A 1+A 3)$. A10, Difference between mixture response and prediction ( $A 8-A 7)$. $A 11$, Difference between response maps evoked by two repeated applications of the mixture. The left color scale applies to difference images $(A 10, A 11)$ and the right color scale to all other images. $B$, Distribution of pixel values in difference images, averaged over $n=18$ experiments with binary amino acid mixtures in which the full set of stimuli was applied twice. Blue, Difference between response map evoked by the mixture and the prediction; cyan, same for a second set of applications; red, difference between response maps evoked by the mixture in repeated applications. $C$, Quantification of the width of distributions of pixel values in difference images. In each of $n=18$ experiments the width of the distribution of pixel values in difference images was measured as the SD of a Gaussian fit. Error bars show the average SD for each type of difference map. The significance of differences in mean SD is shown by symbols in each bar. ${ }^{*} p<0.05 ;{ }^{* *} p<0.01$; ns, not significant. The top row of significance symbols is for comparison between each of the bars to the first (left) bar and the bottom row for comparisons to the second bar. D, Same display as in $A$ for a binary mixture of food extracts. $E$, F, Same plots as in $B, C$ for binary mixtures of food extracts $(n=17)$. Scale bars, $100 \mu \mathrm{m}$.

We next measured afferent glomerular activity in response to different food extracts, each containing many different compounds. These complex stimuli evoked widespread glomerular activity. As a consequence, patterns of $\mathrm{Ca}^{2+}$ signals evoked by different food odor stimuli overlapped greatly (Fig. 2D1-D4). Nevertheless, responses to binary mixtures of food extracts were in large part predictable from the component responses by the algorithm that was used. As for amino acids, lower and upper bounds were similar to each other and to the prediction (Fig. 
2D5-D7). The prediction, but not the sum of the activity maps of the component, was a close approximation of the true response to a binary mixture of food extracts (Fig. 2D7-D11). Similar results with mixtures of food extracts were obtained in $n=24$ experiments.

Quantitative analysis of the deviation of the prediction from the mixture response, as described above, was performed for 17 experiments performed under low-noise conditions (see Materials and Methods). The pixel value differences between prediction and mixture response were not significantly different for repeated series of stimuli ( $p>0.8$; Fig. $2 E$, blue and cyan, $F$ ). As for amino acids, these distributions were slightly but significantly broader than the distributions of differences between repeated mixture responses (Fig. $2 E$, red, $F$ ). The average correlation between the prediction and the mixture response was $0.92 \pm 0.06$ for the first series of stimuli and $0.91 \pm 0.07$ for the second set of stimuli $(p>0.8)$. These values were not significantly different from the mean correlation of responses to repeated mixture applications $(0.95 \pm 0.03 ; p>0.06)$. Hence, as with amino acid stimuli, the correlation between mixture response and prediction was high, and the average difference between the SDs of Gaussian fits was very small $(0.02 \pm 0.05 \% \Delta F / F)$. Thus, responses to binary mixtures of complex food odor stimuli are also predictable within narrow limits by a model assuming no interactions.

Finally, we tested whether the small deviation between the prediction and the response to the mixture depended on the overlap between the component activity patterns. However, the deviation of the mixture from the prediction, quantified by the difference in the width of difference distributions, was not correlated with the correlation between the component activity patterns (amino acids, $r=$ $-0.17 ; p>0.5$; food extracts, $r=-0.19 ; p>0.45$ ). Together, these results indicate that mixture interactions between the tested simple and complex stimuli in the peripheral olfactory system of zebrafish are, at most, weak.

\section{Mitral cell odor responses}

We next examined odor responses of the output neurons of the $\mathrm{OB}$, the MCs. MCs were recorded extracellularly in the loosepatch or cell-attached configuration or were recorded intracellularly by the whole-cell patch-clamp technique. Unlike OSNs, $\mathrm{MCs}$ frequently respond to odors with inhibition. Moreover, MC firing rates often are modulated over tens or hundreds of milliseconds and can comprise successive excitatory and inhibitory epochs. In addition, a subset of mitral cell responses showed subthreshold membrane potential oscillations of $20-30 \mathrm{~Hz}$ (Fig. 3) (Friedrich and Laurent, 2001, 2004). Mitral cell response patterns to the same odor do not change abruptly as a function of concentration and are usually similar over concentration ranges of more than one log unit (R. W. Friedrich, unpublished observations).

The complex response properties arise from synaptic interactions in the $\mathrm{OB}$, indicating that $\mathrm{MC}$ responses do not simply relay their glomerular input but also reflect dynamic processing by neural circuits in the OB. The temporal patterning and the occurrence of inhibitory responses preclude the prediction of mixture responses by simple models of receptor-ligand interactions. We therefore compared MC odor responses by using methods that take into account their temporal response patterns.

Mixture interactions in mitral cell responses: simple odors We first examined responses to amino acids and their binary mixtures. Figure $4 A$ shows responses of one MC, recorded extracellularly. In this example the response to component 1 was clearly excitatory, whereas the response to component 2 was in-
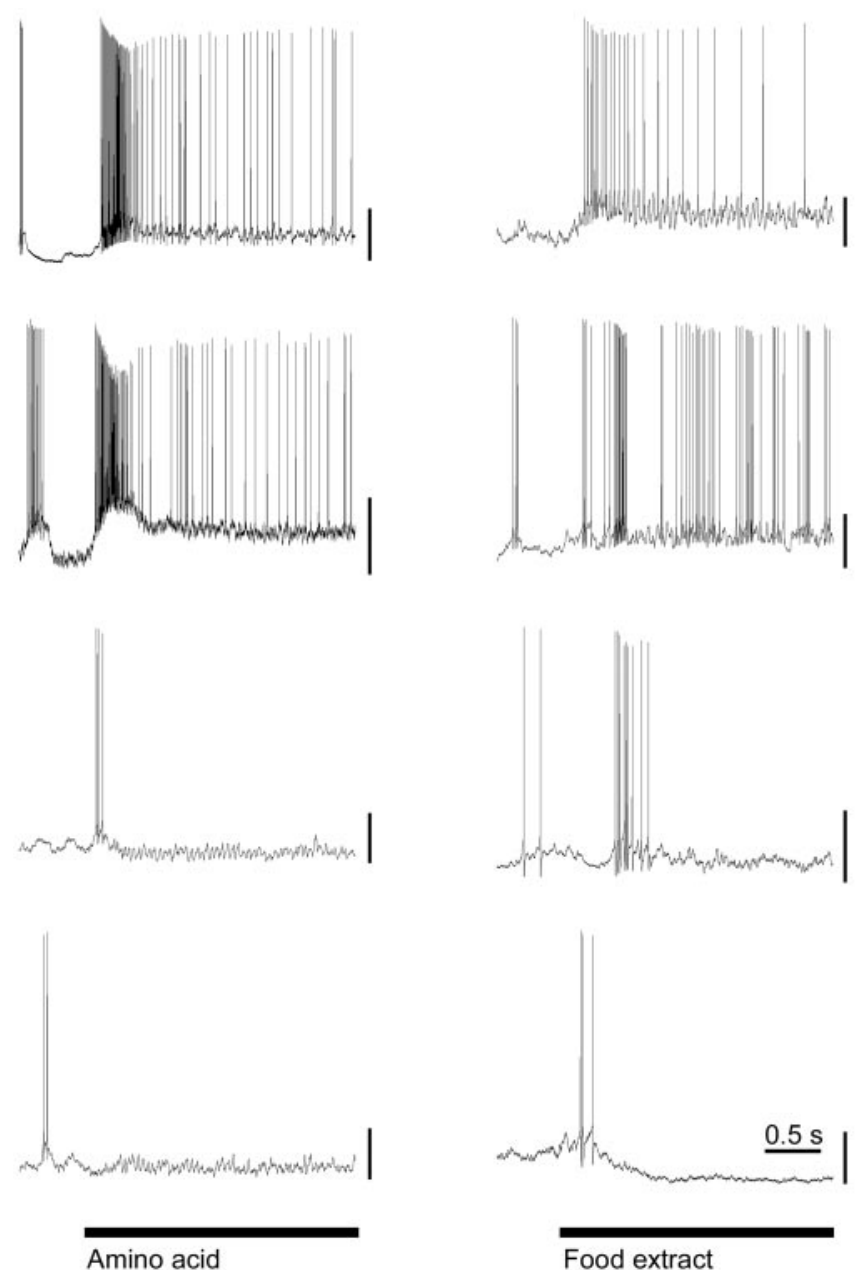

Figure 3. Examples of mitral cell odor responses to amino acids and food extracts. Shown are whole-cell recordings of mitral cell responses to different amino acids (left column) and food extracts (right column). Each response is from a different mitral cell. Both types of stimuli can evoke simple (phasic-tonic; top) and more complex (multi-phasic) response time courses. Fast subthreshold membrane potential oscillations are seen in a subset of odor responses. Odor stimulus is indicated by the horizontal bar. Vertical bars, $20 \mathrm{mV}$.

hibitory. The response to the mixture was excitatory, with a magnitude and time course almost identical to the response to component 1 . Hence, the response to the mixture was dominated by the response to the excitatory component. Figure $4 B$ shows data from another $\mathrm{MC}$ that also responded with clear-cut excitation to one and with clear-cut inhibition to another component. In this case the response to the mixture was dominated by the inhibitory component. In each of 18 experiments testing mixtures of one clearly excitatory and one clearly inhibitory component, one component dominated over the other in the response to the mixture. In $83 \%$ of the cases ( 15 of 18 ) the dominant component was the excitatory one, whereas in the remaining $17 \%$ of the cases $(3$ of 18) the inhibitory component dominated.

In another 18 experiments that used amino acid stimuli, components evoked more complex responses that often differed mainly in their temporal patterns. In the example shown in Figure $4 C$, the responses to both components were excitatory but had different, odor-specific time courses. The response to the mixture was dominated by one of the component responses and was clearly distinct from the response to the other component. A dominance of one component also 
was observed in most of the remaining experiments with components eliciting temporally complex responses.

The same amino acid could elicit a dominant response in one $\mathrm{MC}$ and a nondominant response in another $\mathrm{MC}$, indicating that dominance is not a general property of the stimulus. It was never observed that a MC responded to one or both components, but not to their mixture. In summary, MC responses to binary mixtures of amino acids generally were dominated by one of the components throughout the response. When the component responses were of clearly different sign (excitatory or inhibitory), the mixture response was more often excitatory.

\section{Mixture interactions in mitral cell responses: complex odors}

MC responses to food odors were qualitatively similar to responses evoked by amino acids. They could be simple (mainly excitatory or inhibitory), or they could consist of more complex firing rate modulations, including successive excitatory and inhibitory epochs within the same response (Fig. 3). One difference between responses to simple and complex stimuli was that food odors did not evoke firing rates $>70 \mathrm{~Hz}$, whereas responses to amino acids transiently could reach $>100 \mathrm{~Hz}$ (for example, Fig. $4 B, C)$. This is unlikely to be attributable to lower activation of OSNs by food extracts, because peak $\mathrm{Ca}^{2+}$ signals evoked by food extracts and amino acids in OSN axon terminals were similar.

Responses to binary mixtures of food extracts, measured in 21 experiments, often differed from the responses to both individual components. Figure $5 A$ shows an example of a MC that responded with phasic-tonic excitation to component 1 and with inhibition to component 2 . The response to the mixture was initially excitatory, thus resembling the response to component 1 . However, the initial excitation evoked by the mixture was smaller in magnitude and delayed compared with the excitation evoked by component 1 alone. Subsequently, the response to the mixture became inhibitory and, thus, similar to the response to component 2. Another similar example is shown in Figure $5 B$. The response to a mixture of complex odors therefore is not described accurately by the response to one of the components.

When responses to both components were of the same sign (excitatory or inhibitory) in a given time bin, the sign of the response to the mixture was never of the opposite sign. Hence, the sign of the mixture response corresponded to that of at least one of the component responses in each time bin. However, the mixture response could differ considerably in magnitude from each component response, and the sign of the mixture response could resemble different component responses in different time bins. Responses to binary mixtures of food odors therefore are still related to their component responses, but they are not dom-
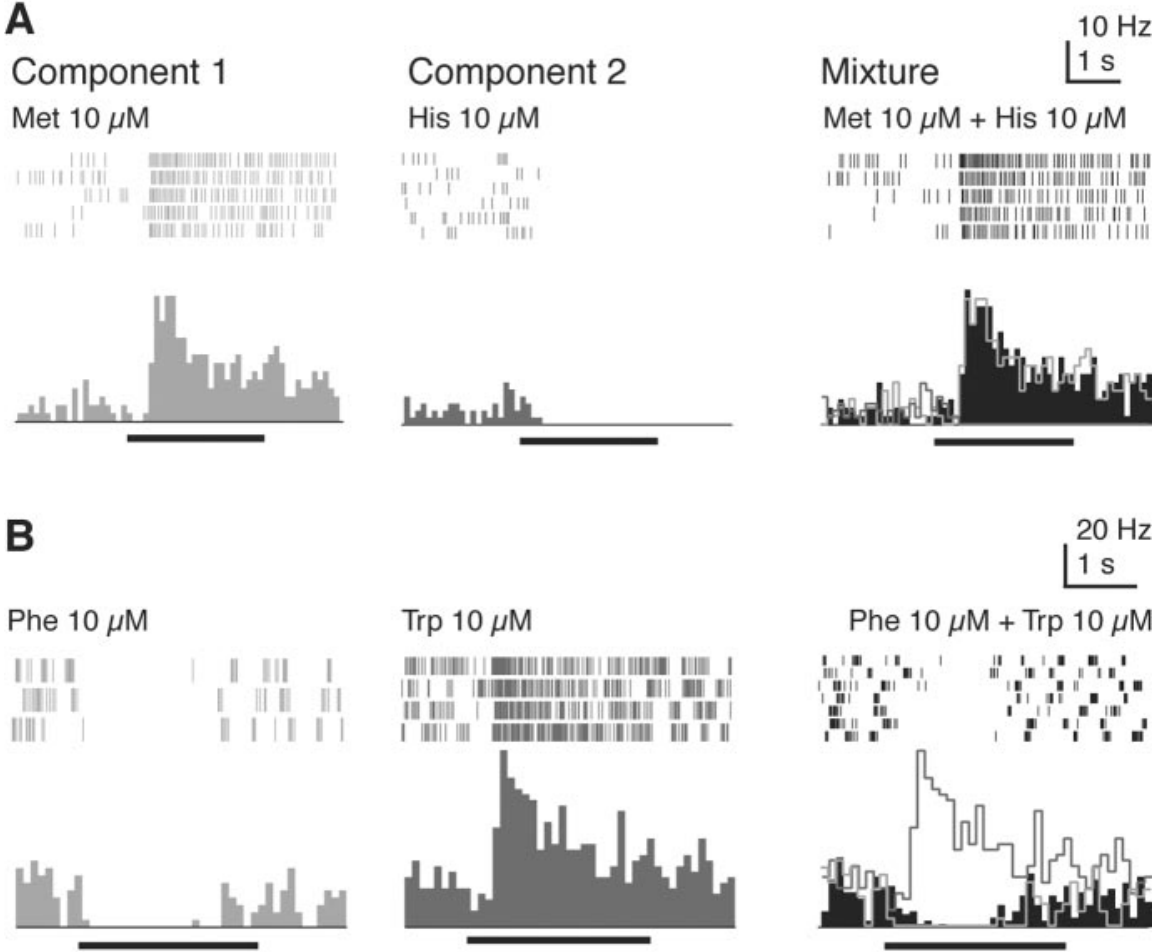

$\operatorname{Trp} 10 \mu \mathrm{M}$

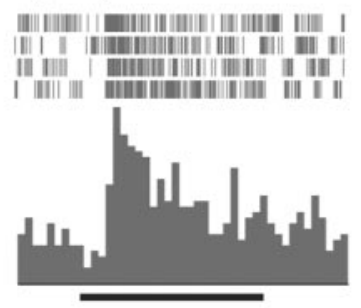

Phe $10 \mu \mathrm{M}+\operatorname{Trp} 10 \mu \mathrm{M}$
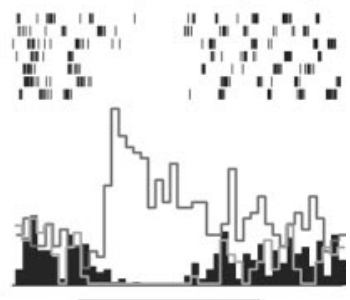

$20 \mathrm{~Hz}$

$1 \mathrm{~s}$

Figure 4. Responses of mitral cells to binary mixtures of amino acids. $A-C$, Responses of three different MCs to different sets of PSTHs show average firing rate in 100 msec time bins. Horizontal bar indicates stimulus presentation. Gray lines in right panels superimpose PSTHs in response to components onto the PSTHs of responses to the mixture.

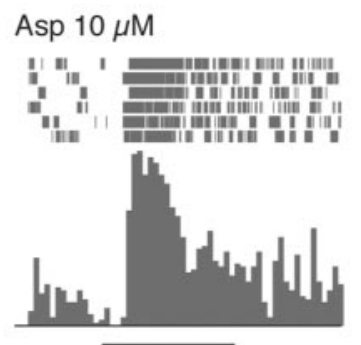

$20 \mathrm{~Hz}$

$1 \mathrm{~s}$

Ala $10 \mu \mathrm{M}+$ Asp $10 \mu \mathrm{M}$
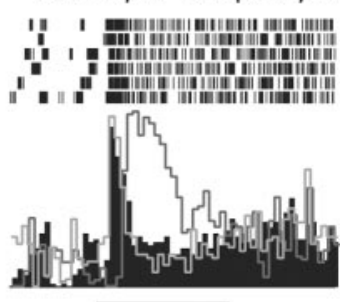

inated as strongly by one of the components as responses to binary amino acid mixtures.

\section{Analysis of component dominance}

The dominance of a component in the response to the mixture was analyzed quantitatively. Each odor response was described by the PSTH of firing rates during the $2.4 \mathrm{sec}$ of odor presentation, averaged over repeated applications, and binned in $100 \mathrm{msec}$ windows. The similarity between the response to the mixture and the responses to the two components was measured by the Pearson correlation coefficient. In each experiment the higher correlation reflects the similarity between the mixture response and the more similar component response. This value therefore was used to quantify component dominance. For binary mixtures of amino acids most component dominance values were high, whereas component dominance values for binary food odor mixtures were distributed more broadly (Fig. 6A). The average component dominance was $0.73 \pm 0.24$ for binary mixtures of amino acids and significantly lower for mixtures of food odors $(0.46 \pm$ 
A

\section{Component 1}

Food extract 1

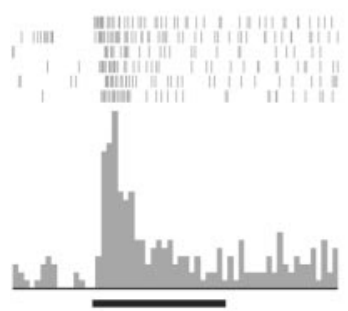

B

Food extract 2

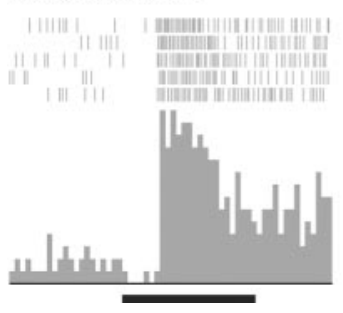

Component 2

Food extract 3
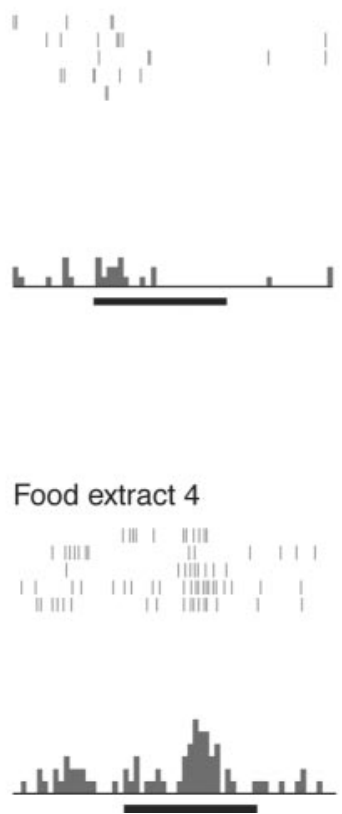

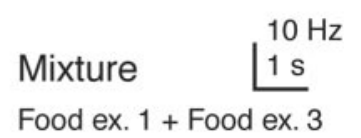

Food ex. $1+$ Food ex. 3

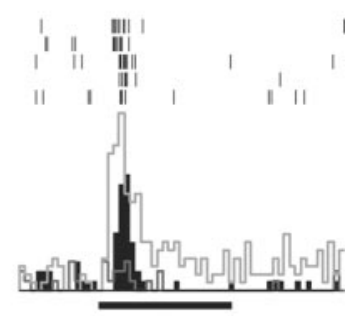

$10 \mathrm{~Hz}$

$1 \mathrm{~s}$

Food ex. 2 + Food ex. 4

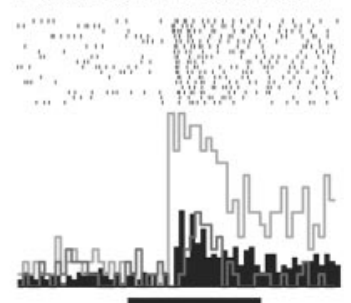

Figure 5. Responses of mitral cells to binary mixtures of food extracts. $A, B$, Responses of two different MCs to different sets of food extracts and their binary mixtures. Action potentials in individual trials and PSTHs ( 100 msec time bins) are shown. Horizontal bar indicates stimulus presentation. Gray lines in right panels superimpose PSTHs in response to components onto the PSTHs of responses to the mixture.

A

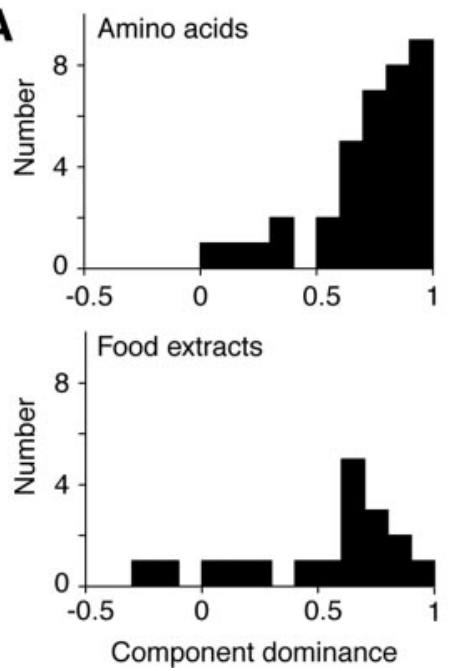

B

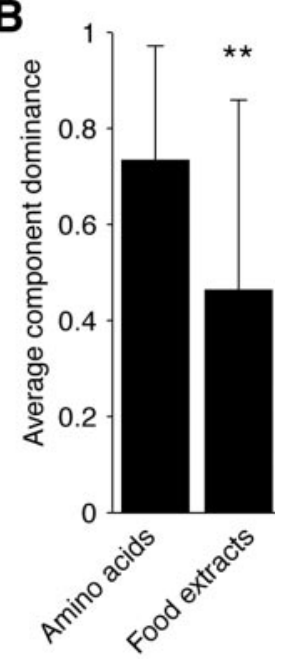

Figure 6. Quantification of component dominance in mitral cell responses to binary mixtures. $A$, Distributions of component dominance values in experiments testing binary mixtures of amino acids (top) and food extracts (bottom). B, Mean component dominance values for binary mixtures of amino acids and food extracts. Error bars show SD; ${ }^{* *} p<0.01$.

$0.40 ; p<0.01$; Fig. $6 B$ ). The average correlation between the nondominant component response and the mixture response was not significantly different for amino acid and food odor stimuli $(-0.10 \pm 0.40$ and $-0.03 \pm 0.40$, respectively; $p>0.5)$. A higher component dominance may be expected if the responses to the individual components are already more similar to each other. However, in our data set the average correlation between the responses to the two components was lower for amino acids

$(-0.10 \pm 0.46)$ than for food odors $(0.17 \pm 0.41)$. We therefore conclude that component dominance is significantly more pronounced in binary mixtures of amino acids.

The correlation coefficient underlying the component dominance measure compares the shapes of PSTHs (i. e., response time courses) independently of the overall firing rate. We therefore also measured the difference in firing rates, averaged over the PSTH, between the responses to each binary mixture and the respective dominant component. The firing rate differences for mixtures of amino acids and food odors were not significantly different from each other (amino acids, $3.8 \pm 4.0 \mathrm{~Hz}$; food odors, $2.4 \pm 2.6 \mathrm{~Hz} ; p>0.1$ ). Hence, for mixtures of food odors the responses to the mixture and those to the dominant component are more different with respect to the time course, but not with respect to the total firing rate.

Finally, we explored whether the component dominance in $\mathrm{MC}$ responses to binary mixtures of amino acids depends on the similarity of afferent activity patterns evoked by the components. The similarity of input patterns was assessed by the correlation between amino acid-evoked activity patterns across OSNs, which were determined previously in zebrafish (Friedrich and Laurent, 2001). As shown in Figure 7, component dominance and the correlation between afferent activity patterns evoked by the components were weakly, but significantly, correlated $(r=0.42 ; p<0.05)$. Hence, component dominance tends to become less pronounced as the overlap of input activity patterns evoked by the components increases.

\section{Discussion}

Mixture interactions in the peripheral olfactory system

Patterns of afferent glomerular $\mathrm{Ca}^{2+}$ signals evoked by binary odor mixtures were predictable within narrow limits from the patterns of the components. The elements of the pattern evoked by the mixture were also active in at least one component response and vice versa. The small differences in response intensities between prediction and mixture response may reflect mixture interactions, the inaccuracy of the prediction, or both. We therefore conclude that mixture interactions in the peripheral olfactory system of zebrafish between the tested odors are weak or negligible. These results are generally consistent with cross-adaptation of glomerular responses in zebrafish (Fuss and Korsching, 2001) and results from intrinsic signal imaging in mice (Belluscio and Katz, 2001).

The $\mathrm{Ca}^{2+}$ indicator selectively reports the activity of sensory inputs to glomeruli. OSNs converging onto the same glomerulus in mice have very similar response properties (Wachowiak et al., 2004), and OSNs respond to odors with simple, stereotyped temporal firing rate patterns in zebrafish (Friedrich and Laurent, $2001,2004)$. It therefore is assumed that individual OSN responses are similar to the glomerular population responses detected by $\mathrm{Ca}^{2+}$ imaging. Indeed, spiking responses of single zebrafish OSNs to mixtures of 16 amino acids were similar to the response to the most potent stimulus alone (Friedrich and Laurent, 2001, 2004), showing 


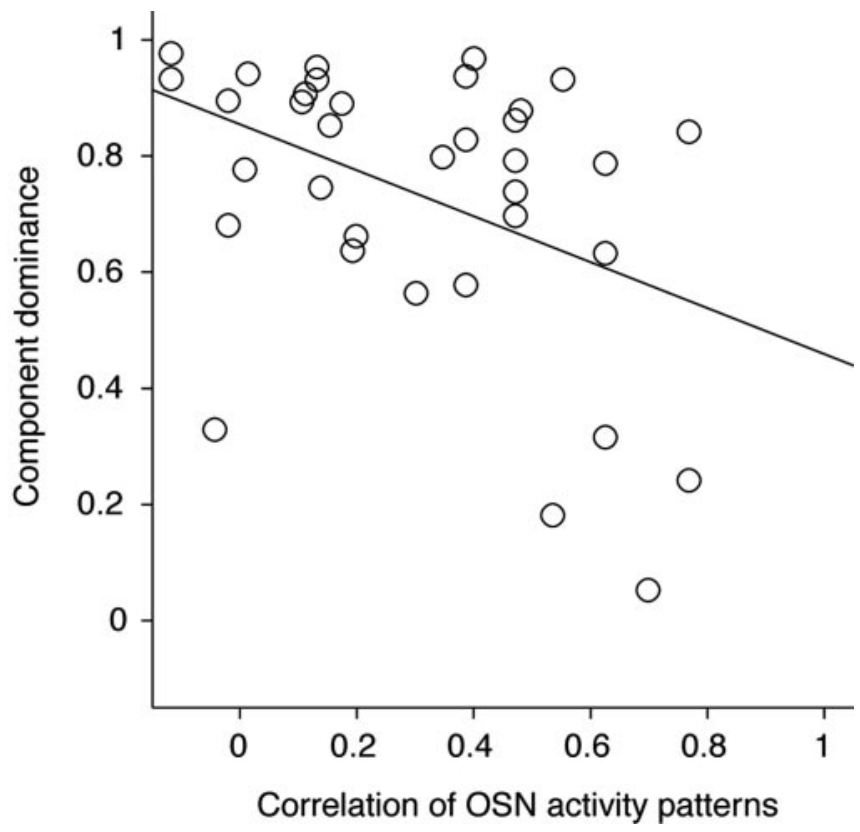

Figure 7. Component dominance as a function of similarity between OSN activity patterns evoked by amino acid components. The correlation between OSN activity patterns evoked by each pair of amino acid components used in the mixture was taken from Friedrich and Laurent (2001) and is plotted on the $x$-axis. The component dominance in mixtures of the same pairs of amino acids is plotted on the $y$-axis. Line shows a linear regression.

that single OSNs responding to at least one component also respond to the mixture, as found for afferent glomerular $\mathrm{Ca}^{2+}$ signals.

It is possible that mixture interactions occur between odorants not tested in this study. Indeed, interactions have been observed between carboxylic acids and amino acids in zebrafish (Fuss and Korsching, 2001) and in OSNs of other species (Ache et al., 1988; Laing et al., 1989; Ache and Zhainazarov, 1995; Kang and Caprio, 1997; Cromarty and Derby, 1998; Spehr et al., 2002). The occurrence of mixture interactions therefore may depend on the odor and species.

In zebrafish it is unknown currently whether each glomerulus is innervated by idiotypic OSNs as in rodents. Activity patterns evoked by mixtures were predicted with little error by an algorithm modeling each afferent glomerular response as a process mediated by a single receptor. This would not be expected if the relation between receptors and glomeruli was different from that in rodents: if populations of OSNs responding to different sets of odors converged onto the same glomerulus, the response to the mixture should be close to the sum of the component responses. If each OSN expressed multiple odorant receptors with different response profiles, the mixture response would become unpredictable. Hence, our data suggest that the functional connectivity between OSNs and glomeruli in the zebrafish $\mathrm{OB}$ is similar to that in rodents.

The glomeruli responding to a mixture were the combination of the glomeruli activated by each component. The density of responding glomeruli therefore should increase with the number of components in a mixture. Indeed, food extracts containing many different compounds evoked widespread and highly overlapping activity patterns. As a consequence, information resides more in the relative response intensity, rather than the identity, of active glomeruli. This may be an important property of representations of natural odor stimuli.
Mixture interactions in the olfactory bulb

Mixture interactions have been observed in the OB of vertebrates (Bell et al., 1987; Kang and Caprio, 1995; Giraudet et al., 2002; Wilson, 2003), but it is unclear whether they arise peripherally or centrally. We found that not all MCs excited by at least one component are also active in response to the mixture. Hence, MC activity patterns evoked by mixtures are not a simple combination of the component patterns, indicating that components interact. Because such interactions were not observed in afferent glomerular activity patterns, they most likely arise in the OB. Possible mechanisms include dendritic integration in MCs and circuit interactions. Because mixture interactions often affect inhibitory and temporally patterned MC responses, circuit interactions are likely candidates (Friedrich and Laurent, 2004).

The component dominance in binary mixtures of single compounds also has been observed in temporally patterned responses of rat MCs (Giraudet et al., 2002). Because it could not be predicted which of the components in a mixture will dominate, the activity pattern across MCs evoked by a binary mixture cannot be predicted accurately from the component patterns. Nevertheless, the pattern evoked by the mixture is related to the component patterns, because the response of each MC resembles the response to one or the other component.

MC responses to binary mixtures of food extracts were dominated less by one component than amino acid mixtures but acquired some novel properties, indicating more complex mixture interactions. Patterns evoked by binary food odor mixtures therefore are related less closely to their component patterns. Each food odor component itself contains many individual compounds. However, at the level of input to the OB, the main difference between food extract and amino acid stimuli is the more widespread and dense activation of glomeruli by food extracts. Differences between mixture interactions among amino acids and food extracts therefore have to be explained by these different properties of glomerular input patterns.

Component dominance decreased with increasing similarity of afferent activity patterns for amino acid stimuli. This trend is consistent with the low component dominance in binary mixtures of food extracts and suggests that mixture interactions become more complex with increasing overlap of afferent component representations. Similar effects have been observed in a simple circuit model of the OB (Linster and Cleland, 2004) and were explained by lateral inhibitory interactions in the $\mathrm{OB}$, which are expected to become denser with increasing pattern overlap. As a consequence, the output of each MC deviates more from its sensory input because the relative strength of inhibitory inputs increases. Hence, the dependence of component dominance on the overlap of input activity patterns may reflect nonlinear interactions mediated by inhibitory interneurons in the OB. However, additional insights are required to understand the mechanisms underlying mixture interactions in the OB.

Mixture interactions also have been described at the next level of the olfactory pathway. These mixture interactions are experiencedependent and appear to contribute to the establishment of complex receptive fields of neurons in the anterior piriform cortex (Wilson, 2003). Mixture interactions in higher olfactory areas therefore also may contribute to the processing and the synthetic perception of odor mixtures (Wilson and Stevenson, 2003).

\section{Mixture representations in the olfactory bulb and odor perception}

Our results suggest tentative rules describing some properties of binary mixture interactions in the $\mathrm{OB}$ that are similar to conclu- 
sions derived from the model by Linster and Cleland (2004). First, patterns of glomerular input activity evoked by a mixture are given by the combination of the patterns of the components. Second, MC responses to mixtures of dissimilar compounds are dominated quantitatively by the response to one of the components. Third, for mixtures of similar components, the sign, but not necessarily the magnitude, of the response corresponds to that of at least one of the components. Additional properties of mixture interactions may exist. For example, it is possible that the relative strength of components influences mixture interactions.

The properties of mixture interactions in the OB lead to predictions concerning odor representations by activity patterns across multiple glomeruli or MCs. First, the predictability of a mixture representation across glomeruli from the representations of the components is high. Much of the information about the components is therefore, in theory, available at the level of glomerular inputs. Second, the predictability of mixture representations by MCs is generally lower than the predictability of afferent mixture representations, indicating that processing in the OB discards component information. This may contribute to the synthetic nature of odor perception and constrain the analytical power of olfactory processing. Third, MC representations of mixtures of simple dissimilar components combine parts of the representation of each component, because the response of each $\mathrm{MC}$ represents one or the other component. Hence, the mixture representation neither is close to the average of the representations of the components nor is it a novel representation, but the response of each $\mathrm{MC}$ is predictable with a certain probability. This organization retains some information about the components. Fourth, with increasing similarity of the components the representation of the mixture is still related to those of the components but becomes less predictable. Information about the components therefore decreases, and the representation acquires novel properties. This effect is pronounced for mixtures of food extracts, suggesting that mixture interactions play an important role in the processing of natural stimuli. One function of mixture interactions also may be to limit the total MC activity when the number of active glomeruli increases.

Our results give rise to experimentally approachable predictions about the effect of mixture interactions on odor perception that can be compared with existing psychophysical or behavioral data from other vertebrates. The component information in the representation of a mixture should decrease with the number of components, because fewer responses of MCs should be dominated by each component and because component dominance should decrease in general. Indeed, human psychophysics demonstrated that the segmentation of a mixture into its components becomes impossible when the number of components exceeds 3-4 (Laing and Francis, 1989; Livermore and Laing, 1996). Furthermore, component identification should become more difficult and the perception of a binary mixture should acquire a more novel quality with increasing similarity of the components. Indeed, rats cannot identify components reliably in binary mixtures of similar, but not dissimilar, compounds. Rather, mixtures of similar compounds are perceived as different from both components (Wiltrout et al., 2003). Hence, mixture interactions in the OB may play an important role in odor perception.

\section{References}

Ache BW, Zhainazarov A (1995) Dual second-messenger pathways in olfactory transduction. Curr Opin Neurobiol 5:461-466.

Ache BW, Gleeson RA, Thompson HA (1988) Mechanisms for mixture suppression in olfactory receptors of the spiny lobster. Chem Senses 13:425-434.

Baier H, Korsching S (1994) Olfactory glomeruli in the zebrafish olfactory system form an invariant pattern and are identifiable across animals. J Neurosci 14:219-230.

Bell GA, Laing DG, Panhuber H (1987) Odour mixture suppression: evidence for a peripheral mechanism in human and rat. Brain Res 426:8-18.

Belluscio L, Katz LC (2001) Symmetry, stereotypy, and topography of odorant representations in mouse olfactory bulbs. J Neurosci 21:2113-2122.

Byrd CA, Brunjes PC (1995) Organization of the olfactory system in the adult zebrafish: histological, immunohistochemical, and quantitative analysis. J Comp Neurol 358:247-259.

Carr WES (1988) The molecular nature of chemical stimuli in the aquatic environment. In: Sensory biology of aquatic animals (Atema J, Fay RR, Popper AN, Tavolga WN, eds), pp 3-27. New York: Springer.

Cromarty SI, Derby CD (1998) Inhibitory receptor binding events among the components of complex mixtures contribute to mixture suppression in responses of olfactory receptor neurons of spiny lobsters. J Comp Physiol [A] 183:699-707.

Derby CD, Ache BW, Kennel EW (1985) Mixture suppression in olfaction: electrophysiological evaluation of the contribution of peripheral and central neural compartments. Chem Senses 10:301-316.

Derby CD, Hutson M, Livermore BA, Lynn WH (1996) Generalization among related complex odorant mixtures and their components: analysis of olfactory perception in the spiny lobster. Physiol Behav 60:87-95.

Edwards JG, Michel WC (2002) Odor-stimulated glutamatergic neurotransmission in the zebrafish olfactory bulb. J Comp Neurol 454:294-309.

Friedrich RW, Korsching SI (1997) Combinatorial and chemotopic odorant coding in the zebrafish olfactory bulb visualized by optical imaging. Neuron 18:737-752.

Friedrich RW, Korsching SI (1998) Chemotopic, combinatorial, and noncombinatorial odorant representations in the olfactory bulb revealed using a voltage-sensitive axon tracer. J Neurosci 18:9977-9988.

Friedrich RW, Laurent G (2001) Dynamic optimization of odor representations in the olfactory bulb by slow temporal patterning of mitral cell activity. Science 291:889-894.

Friedrich RW, Laurent G (2004) Dynamics of olfactory bulb input and output activity during odor stimulation in zebrafish. J Neurophysiol 91:2658-2669.

Fuss SH, Korsching SI (2001) Odorant feature detection: activity mapping of structure response relationships in the zebrafish olfactory bulb. J Neurosci 21:8396-8407.

Giraudet P, Berthommier F, Chaput M (2002) Mitral cell temporal response patterns evoked by odor mixtures in the rat olfactory bulb. J Neurophysiol 88:829-838.

Kang JS, Caprio J (1995) Electrophysiological responses of single olfactory bulb neurons to binary mixtures of amino acids in the channel catfish, Ictalurus punctatus. J Neurophysiol 74:1435-1443.

Kang JS, Caprio J (1997) In vivo responses of single olfactory receptor neurons of channel catfish to binary mixtures of amino acids. J Neurophysiol $77: 1-8$.

Laing DG, Francis GW (1989) The capacity of humans to identify odors in mixtures. Physiol Behav 46:809-814.

Laing DG, Willcox ME (1983) Perception of components in binary mixtures. Chem Senses 7:249-264.

Laing DG, Panhuber H, Willcox ME, Pittman EA (1984) Quality and intensity of binary odor mixtures. Physiol Behav 33:309-319.

Laing DG, Cain WS, McBride RL, Ache BW (1989) Perception of complex smells and tastes. San Diego: Academic.

Lancet D, Greer CA, Kauer JS, Shepherd GM (1982) Mapping of odorrelated neuronal activity in the olfactory bulb by high-resolution 2-deoxyglucose autoradiography. Proc Natl Acad Sci USA 79:670-674.

Linster C, Cleland TA (2004) Configurational and elemental odor mixture perception can arise from local inhibition. J Comput Neurosci 16:39-47.

Livermore A, Laing DG (1996) Influence of training and experience on the perception of multicomponent odor mixtures. J Exp Psychol Hum Percept Perform 22:267-277.

Malnic B, Hirono J, Sato T, Buck LB (1999) Combinatorial receptor codes for odors. Cell 96:713-723.

Mathieson WB, Maler L (1988) Morphological and electrophysiological 
properties of a novel in vitro preparation: the electrosensory lateral line lobe brain slice. J Comp Physiol [A] 163:489-506.

Mombaerts P (1999) Seven-transmembrane proteins as odorant and chemosensory receptors. Science 286:707-711.

Moskowitz HR, Barbe CD (1977) Profiling of odor components and their mixtures. Sens Processes 1:212-226.

Ressler KJ, Sullivan SL, Buck LB (1994) Information coding in the olfactory system: evidence for a stereotyped and highly organized epitope map in the olfactory bulb. Cell 79:1245-1255.

Rubin BD, Katz LC (1999) Optical imaging of odorant representations in the mammalian olfactory bulb. Neuron 23:499-511.

Spehr M, Wetzel CH, Hatt H, Ache BW (2002) 3-Phosphoinositides modulate cyclic nucleotide signaling in olfactory receptor neurons. Neuron 33:731-739.

Staubli U, Fraser D, Faraday R, Lynch G (1987) Olfaction and the "data" memory system in rats. Behav Neurosci 101:757-765.

Stewart WB, Kauer JS, Shepherd GM (1979) Functional organization of rat olfactory bulb analysed by the 2-deoxyglucose method. J Comp Neurol 185:715-734.

Valentincic T, Kralj J, Stenovec M, Koce A, Caprio J (2000) The behavioral detection of binary mixtures of amino acids and their individual components by catfish. J Exp Biol 203 [Pt 21]:3307-3317.

Vassar R, Chao SK, Sitcheran R, Nunez JM, Vosshall LB, Axel R (1994) Topographic organization of sensory projections to the olfactory bulb. Cell 79:981-991.

Wachowiak M, Cohen LB (2001) Representation of odorants by receptor neuron input to the mouse olfactory bulb. Neuron 32:723-735.

Wachowiak M, Denk W, Friedrich RW (2004) Functional organization of sensory input to the olfactory bulb glomerulus analyzed by two-photon calcium imaging. Proc Natl Acad Sci USA 101:9097-9102.

Wilson DA (2003) Rapid, experience-induced enhancement in odorant discrimination by anterior piriform cortex neurons. J Neurophysiol 90:65-72.

Wilson DA, Stevenson RJ (2003) The fundamental role of memory in olfactory perception. Trends Neurosci 26:243-247.

Wiltrout C, Dogra S, Linster C (2003) Configurational and nonconfigurational interactions between odorants in binary mixtures. Behav Neurosci 117:236-245.

Zhang X, Firestein S (2002) The olfactory receptor gene superfamily of the mouse. Nat Neurosci 5:124-133. 